\title{
Speciation: Determination of Methylmercury in Fish Samples with HPLC-ICP-MS
}

\author{
S. Ghanthimathi*a, Norhidayu bt Ibrahim ${ }^{a}$, Zalilah bt Nasir ${ }^{a}$, Lilian Lim ${ }^{\text {b }}$, and Kenneth Ong \\ a Metal Contaminant Unit, National Public Health Laboratory, Sungai Buloh, Malaysia \\ b PerkinElmer Malaysia \\ c PerkinElmer Singapore
}

\section{INTRODUCTION}

Several forms of mercury (Hg) occur naturally in the environment, the most common being metallic mercury, mercuric sulfide (cinnabar ore), mercuric chloride, and methylmercury.

Methylmercury is the predominant mercury species in fish. The US Environmental Protection Agency (EPA) states in an updated mercury overview paper that in most adult fish, 90 to $100 \%$ of mercury content is methylmercury (US EPA, 2001).

Methylmercury is also a welldocumented neurotoxin, which may cause adverse effects on the developing brain. This compound passes both the placental barrier and the blood-brain barrier; as such, exposures during pregnancy are the main concern. Additionally, the overall evaluation for methylmercury compounds is important since they are carcinogenic to humans.

The compound dealt with most extensively in toxicological research in recent years is methylmercury. Generally, organic forms are much more toxic than the inorganic forms. Mercury is present in fish and marine mammals consumed by humans worldwide. Methylmercury is formed naturally (from anthropogenic sources and naturally released mercury) by biological activity in aquatic environments, and is bio-magnified in the food chain, resulting in much higher concentrations in higher predatory

${ }^{*}$ Corresponding author.

E-mail: ghanthimathi@moh.gov.my

\section{ABSTRACT}

Two mercury species in National Research Council of Canada Certified Reference Material (NRCC CRM) (a) TORT-2 and (b) DORM-2 can be determined using high performance liquid chromatography (HPLC) in conjunction with inductively coupled plasma mass spectrometry (ICP-MS). A non-aggressive extraction method was employed to maintain the integrity of the species in the samples.

Chromatographic separation of mercury species could be achieved in 6 minutes with a reversed phase column using $0.1 \%$ of L-cysteine as the mobile phase. The limit of detection for this method is $0.5 \mathrm{ppb}(\mu \mathrm{g} / \mathrm{kg})$, sufficient to determine low levels of mercury in fish samples.

The accuracy of the method was evaluated by analyzing marine biological certified reference materials (TORT-2 and DORM-2). The proposed method was then successfully applied to the determination of methylmercury compounds in "Ikan Kembung", a popular local fish for grilling, also known as Indian Mackerel.

fish and mammals than in water and lower organisms. Most of the total mercury concentrations in fish are in the form of methylmercury (close to $100 \%$ for older fish).

Methylmercury has been found to have adverse effects on several organ systems in the human body as well as in animals. These include the nervous system (mental retardation, deafness, blindness, impairment of speech, etc.), the kidneys, the blood and the heart system (blood pressure, heart-rate variety and heart diseases). Other possible effects include cancer and genotoxicity. From research done on animals, there is evidence on its adverse effects on the immune system and the reproductive system. Methylmercury compounds, as a whole, are classified as possibly carcinogenic to humans.

In order to carry out the proposed studies, it was necessary to select the proper method for the determination of the methylmercury content in fish which would meet the criteria of modern analytical methodology. The method had to have good reproducibility, repeatability, and a high degree of recovery to ascertain good accuracy.

The methodology applied was based on extracting methylmercury with toluene in an acidic medium. The extract was then back-extracted into aqueous phase with L-cysteine hydrochloride anhydrous (L-cysteine.HCl). The methylmercury content in the aqueous extract was determined using HPLC-ICP-MS.

In general, speciation of mercury species involves the following steps:

(a) Sample collection / pretreatment / preservation / storage

(b) Extraction of mercury from the matrix / clean-up / preconcentration

(c) Separation of mercury species of interest

(d) Detection 


\section{Regulations}

The recognition of methylmercury toxicity and the realization that fish are the major source of human exposure has led to the development of legislation by governments and health organizations throughout the world.

The majority of countries and global organizations now enforce maximum concentrations of mercury in fish to approximately $0.5 \mathrm{mg} / \mathrm{kg}$ wet weight.

The European Commission (EC) has introduced the EC 1881/2006 regulation, setting maximum levels for certain contaminants in foodstuffs. With regards to mercury in fishery products and muscle meat of fish, the rule mandates a maximum concentration of $0.5 \mathrm{mg} / \mathrm{kg}$ wet weight.

In order to comply with these stringent regulations, governments and health organizations need a reliable method for the analysis of mercury in fish. High performance liquid chromatography inductively coupled plasma mass spectrometry (HPLC-ICP-MS) is a perfect tool for the measurement of low levels of mercury in fish. An experiment was developed to demonstrate the capa-

TABLE I ICP-MS Operating Conditions and Performance

\begin{tabular}{ll}
\hline Instrument & ELAN DRC-e ICP-MS \\
Analytes & Hg 199.968 amu \\
Calibration & External \\
RF Power & $1300 \mathrm{~W}$ \\
RPa Setting & 0 \\
RPq Setting & 0.25 \\
Mode & Standard \\
Dwell Time & $250 \mathrm{~ms}$ \\
Sweeps / Reading & 1 \\
Reading/ Replicates & 3557 \\
Replicates & 1 \\
Spray Chamber & Cyclonic Spray Chamber \\
Nebulizer & Meinhard Nebulizer \\
\hline
\end{tabular}

bility of HPLC-ICP-MS to achieve precise, dependable analysis of low levels of mercury in fish.

\section{EXPERIMENTAL}

\section{ICP-MS Operating Conditions}

For speciation analysis, detection of the HPLC eluent was accomplished with a PerkinElmer ${ }^{\circledR}$ ELAN $^{\circledR}$ DRC-e ICP-MS instrument (PerkinElmer, Inc., Shelton, CT, USA). The operating conditions of the ICP-MS and the data acquisition parameters are listed in Table I.

\section{Chromatographic Operating Conditions}

The HPLC apparatus was equipped with a PerkinElmer ${ }^{\circledR}$ Series 200 quaternary HPLC pump, autosampler, vacuum degasser, and Peltier column oven. All separations were performed at room temperature under isocratic conditions. The column used for this separation was a PerkinElmer ${ }^{\circledR} \mathrm{C}_{18}$ aqueous column, $150 \mathrm{~mm}$ in length, with a $4.6-\mathrm{mm}$ ID and $5-\mu \mathrm{m}$ parti- cles. Sample aliquots of $50 \mu \mathrm{L}$ aliquots were injected using a 200- $\mu \mathrm{L}$ PEEK injection loop.

The coupling of HPLC to ICP-MS was accomplished by directing the eluant from the column to the switching valve. The sample was then transported to the ICP-MS for mercury determination. The optimal experimental conditions are listed in Table II.

\section{Reagents}

HPLC Mobile Phase Preparation

The HPLC mobile phase $(0.1 \%$ (w/v) L-cysteine) was prepared by dissolving $1 \mathrm{~g}$ of L-cysteine in $1 \mathrm{~L}$ of deionized water.

\section{Gold Standard as Wash Solution Preparation}

$500 \mathrm{ppb}$ of gold standard was prepared from a 1000-ppm (mg/L) gold (PerkinElmer) standard solution in deionized water. This was injected as wash solution in between all of the samples and standards.

\section{TABLE II}

HPLC Operating Conditions and Performance

\begin{tabular}{|c|c|}
\hline Instrument & $\begin{array}{l}\text { HPLC Series } 200 \text { with Autosampler, Peltier } \\
\text { Column Oven, Quaternary Pump, Vacuum } \\
\text { Degasser and Rheodyne }{ }^{\circledR} \text { Switching Valve }\end{array}$ \\
\hline Software & Chromera Software Version 1.2 \\
\hline Column & $\begin{array}{l}\text { PerkinElmer } \mathrm{C}_{18} \text { Aqueous } \\
\text { (a) Column Length: } 150 \mathrm{~mm} \\
\text { (b) Inside Diameter: } 4.6 \mathrm{~mm} \\
\text { (c) Particle Size: } 5 \mu \mathrm{m} \\
\text { (d) Pore Size: } 100 \AA\end{array}$ \\
\hline Injection Volume & $50 \mu \mathrm{L}$ \\
\hline Oven Temperature & $25^{\circ} \mathrm{C}$ \\
\hline Pump & Isocratic \\
\hline Mobile Phase & $0.1 \% \mathrm{w} / \mathrm{v}$ L-cysteine \\
\hline Flow Rate & $1 \mathrm{~mL} / \mathrm{min}$ \\
\hline Sample Loop & $200 \mu \mathrm{L}$ \\
\hline LC Pump Program & $\begin{array}{l}\text { (a) Step } 0=1 \mathrm{~min} \text { (Equilibrium): } 100 \% \text { of } 0.1 \% \\
\text { (w/v) L-cysteine with flow rate of } 1 \mathrm{~mL} / \mathrm{min} \\
\text { (b) Step } 1=6 \text { min (Run): } 100 \% \text { of } 0.1 \% \mathrm{w} / \mathrm{v} \\
\text { L-cysteine with flow rate of } 1 \mathrm{~mL} / \mathrm{min} \\
\text { (c) Step } 2=1 \mathrm{~min} \text { (Wash): } 100 \% \text { of } 0.1 \%(\mathrm{w} / \mathrm{v}) \\
\text { L-cysteine with flow rate of } 1 \mathrm{~mL} / \mathrm{min}\end{array}$ \\
\hline
\end{tabular}




\section{Standard Preparation}

The calibration blank $(0.1 \%$ (w/v) L-cysteine. $\mathrm{HCl}$ ) was prepared by dissolving $1 \mathrm{~g}$ of L-cysteine. $\mathrm{HCl}$ in $1 \mathrm{~L}$ of deionized water.

Mercury standards were prepared from a $1000-\mathrm{ppm}(\mathrm{mg} / \mathrm{L})$ mercury (PerkinElmer) standard solution in $0.1 \%(\mathrm{w} / \mathrm{v}) \mathrm{L}-\mathrm{cysteine} \cdot \mathrm{HCl}$.

Methylmercury chloride $\left(\mathrm{CH}_{3} \mathrm{HgCl}\right)$ was supplied by Fluka, and a stock solution of $2000 \mathrm{ppm}$ (mg/L) $\mathrm{Hg}$ was prepared by dissolving $\mathrm{CH}_{3} \mathrm{HgCl}$ in $0.1 \%$ (w/v) L-cysteine $\cdot \mathrm{HCl}$. A suitable amount of 2-mercaptoethanol (Sigma) was added in methylmercury chloride standard to help in dissolving.

These standards were first diluted to produce a $10-\mathrm{ppm}$ (mg/L) stock solution to allow simple preparation of a range of standards. Standards of different mercury concentrations were made by appropriate dilution of the intermediate standards with $0.1 \%(\mathrm{w} / \mathrm{v})$ L-cysteine.HCl.

All stock standard solutions were protected from light and stored at $4{ }^{\circ} \mathrm{C}$. The mercury working standards were prepared daily by proper dilution with $0.1 \%(\mathrm{w} / \mathrm{v})$ L-cysteine $\cdot \mathrm{HCl}$.

For method development, two certified reference materials (CRMs) were used as test samples: Lobster
Hepatopancreas Reference Material for Trace Metals (TORT-2) and Dogfish Muscle Certified Reference Material for Trace Metals from National Research Council Canada (NRCC).

\section{Sample Preparation}

A 5-g amount of freeze-dried sample (Figure 1) was weighed into a 50-mL polypropylene (PP) tube, followed by the addition $10 \mathrm{~mL}$ of toluene. The content in the PP tube was stirred for 5 minutes with a vortex mixer to disperse the sample homogeneously and to ensure that all methylmercury dissolved in toluene. Next, another $25 \mathrm{~g}$ of $0.1 \%$ L-cysteine $\cdot \mathrm{HCl}$ was added as backextraction solution. The tube was shaken to extract all forms of mercury into the aqueous phase.

The sample tube was centrifuged at $800 \mathrm{rpm}$ for 3 minutes to separate the toluene layer from the aqueous layer. By using a plastic syringe with a needle, the aqueous

\section{A $_{\text {Spectroscopy }}^{\text {tomic }}$ \\ Vol. 32(3), May/June 2011}

(bottom) layer was collected and transferred to another clean PP tube. The tube with only the aqueous phase was centrifuged again at $4000 \mathrm{rpm}$ for 5 minutes.

The solutions were transferred to the HPLC vials using a plastic syringe with a polyvinylidene fluoride (PVDF) syringe filter attached. For analysis, an aliquot of this extract was injected into the HPLCICP-MS system.

\section{RESULTS AND DISCUSSION}

Typical chromatograms for a standard containing $2 \mathrm{ppb}$ each of inorganic mercury $(\mathrm{Hg})$ and methylmercury (MtHg) and the DORM-2 CRM are shown in Figure 2. As evidenced from these chromatograms, the species are fully resolved and separation is achieved in less than 6 minutes. Figure 2 also shows that the DORM matrix does not affect the chromatography since the peaks from the SRM and the standards elute at the same time.

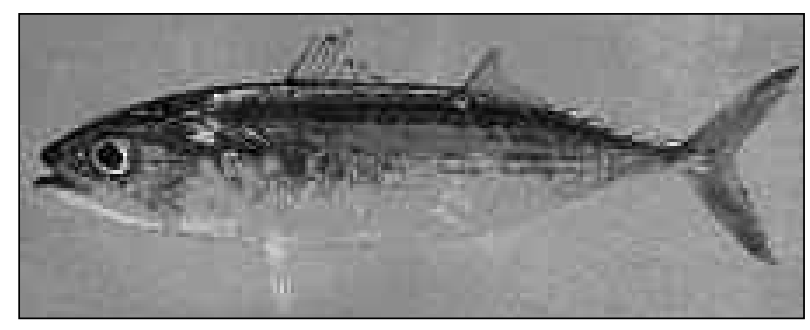

Fig. 1. Indian Mackerel fish.

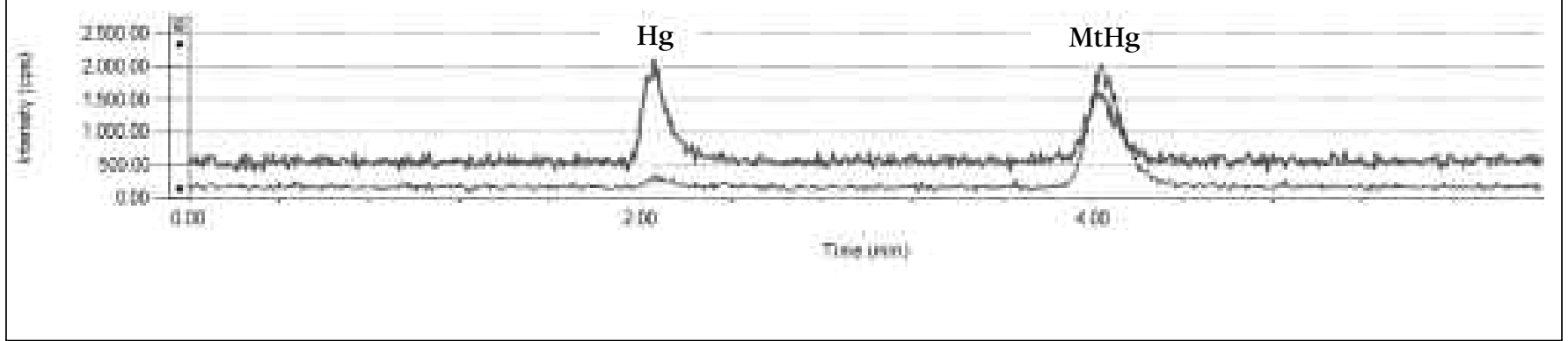

Fig. 2. Chromatograms of a standard containing 2 ppb of inorganic mercury and methylmercury (upper trace) and of the CRM DORM-2 (lower trace). 
An initial test of the stability of the method was performed by making replicate injections of the $2 \mathrm{ppb}$ standard and overlaying the chromatograms, as shown in Figure 3. The near perfect overlap of the chromatograms demonstates the repeatability of the method.

This method was able to detect low levels of mercury in fish samples at a limit of detection (LOD) of $0.5 \mathrm{ppb}(\mu \mathrm{g} / \mathrm{kg})$, as determined by injecting successively lower concentrations until the peaks were three times the amplitude of the baseline noise. The calibration curve also showed excellent linearity starting from $0.5 \mathrm{ppb}$ up to $6 \mathrm{ppb}$ with a correlation coefficient $\left(R^{2}\right)$ value of $>0.995$. Figure 4 shows the chromatograms of the calibration standards. This linearity demonstrates the superb performance of HPLCICP-MS over a wide concentration range.

In order to validate the HPLCICP-MS method, mercury compounds were determined in the TORT-2 and DORM-2 reference sample. These two CRMs were spiked with 4 ppb mercury and 2 ppb methylmercury prior to extraction and compared with unspiked samples to calculate the recoveries.

The repeatability of the method was validated by conducting 3 parallel determinations (CRM, Spike 1 and 2) of mercury with a certified value of $0.27 \pm 0.06 \mathrm{mg} / \mathrm{kg}$ and methylmercury with a certified value of $0.152 \pm 0.013 \mathrm{mg} / \mathrm{kg}$ in TORT-2.

The accuracy of the method was also determined on the basis of the analysis extracts from the certified reference material (DORM-2) which contained high levels of total mercury with a certified value of $4.64 \pm 0.26 \mathrm{mg} / \mathrm{kg}$ and methylmercury with a certified value of $4.47 \pm 0.32 \mathrm{mg} / \mathrm{kg}$. Due to the high level of methylmercury in DORM-2, the extracted solution was further diluted by another 25 times to avoid carry-over effects. As such, only methylmercury was determined since the inorganic content

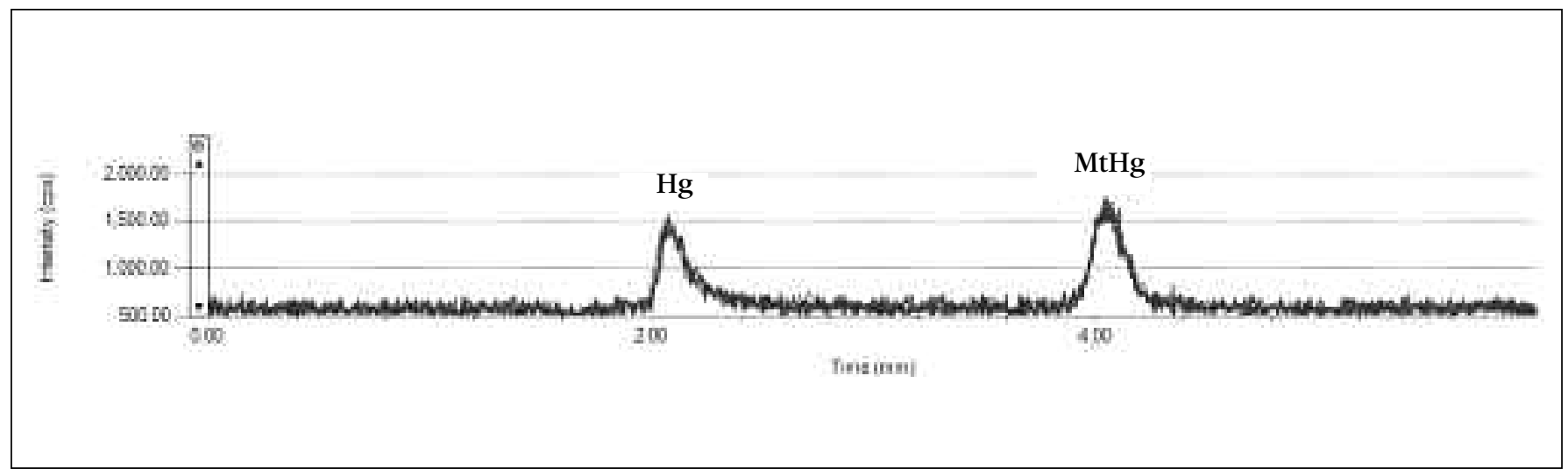

Fig. 3. Consecutive injections of a 2-ppb standard, demonstrating repeatability.

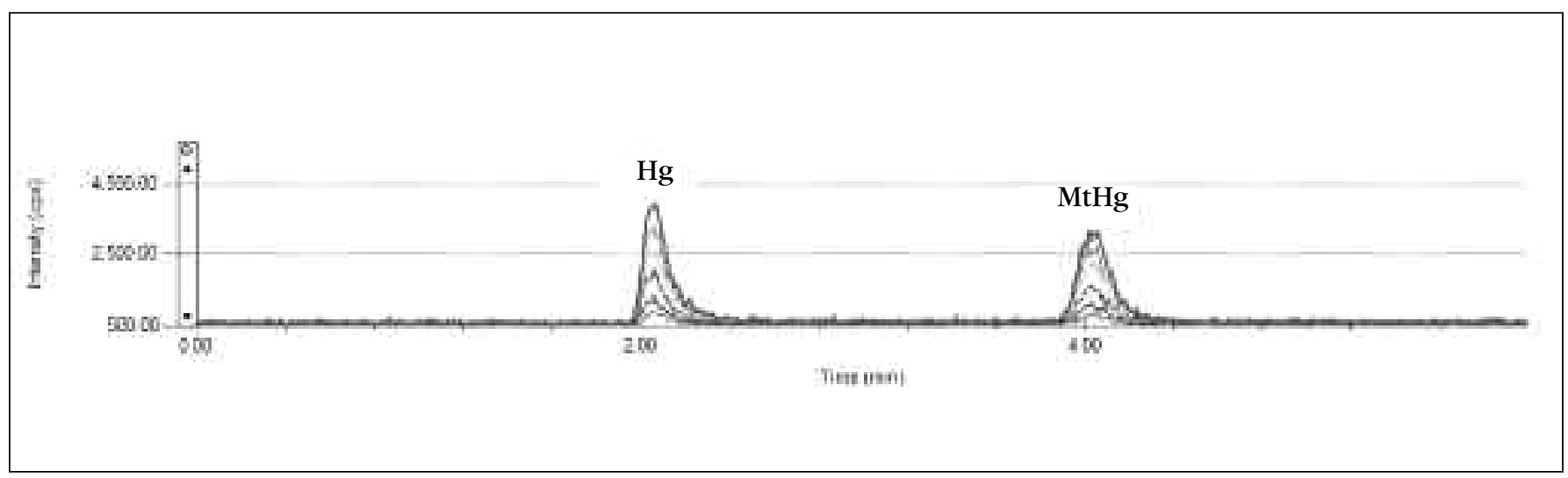

Fig. 4. Chromatograms of the calibration standards: $0.5-6.0 \mathrm{ppb} \mathrm{Hg} / \mathrm{MtHg}$. 
$(0.47 \mathrm{mg} / \mathrm{kg})$ was below the detection level after the large dilution factors and thus not reported (as can be seen in Figure 2).

The values determined were compared with the certified values. The recovery of the applied method was between $\pm 10 \%$ for mercury and $\pm 20 \%$ for methylmercury (see Table III).

To further validate the efficiency of the extraction method based on methylmercury only in the Indian Mackerel fish samples, the samples with 6 parallel determinations were spiked with 2 ppb methylmercury prior to extraction and compared with unspiked samples in order to calculate the recoveries.

No inorganic mercury was detected in Mackerel Fish 399, while methylmercury was detected at $\sim 0.4 \mathrm{ppb}$. The methylmercury spike recoveries for Indian Mackerel Fish are shown in Table IV. Agreement with the expected results was good for methylmercury, with the recovered values all falling within $\pm 20 \%$ of the expected values.

\section{CONCLUSION}

This work has demonstrated the ability to determine mercury species by using the combination of high performance liquid chromatography (HPLC) and inductively coupled plasma mass spectrometry (ICP-MS). The method offers excellent limits of detection (LOD), linear range, stability, and accuracy for the determination of trace levels of mercury species in fish.

In this study, a simple pretreatment method was developed for the extraction of mercury compounds in fish samples. The superb sensitivity, precision, and excellent detection limits of this method easily meet the levels required by the EU Commission regulations. The speed and efficiency of HPLC-ICP-MS allow a sample to be analyzed approximately every 6 minutes.
TABLE III

Recovery for Certified Reference Material TORT-2 and DORM-2

\begin{tabular}{lrr}
\hline Sample ID & \multicolumn{2}{c}{ Recoveries } \\
& $\mathrm{Hg}$ & \multicolumn{1}{c}{ MtHg } \\
\hline DORM-2 Diluted & $\mathrm{NA}$ & $89.7 \%$ \\
TORT-2 & $117.8 \%$ & $100.9 \%$ \\
CRM Spike 1 & $108.1 \%$ & $85.1 \%$ \\
CRM Spike 2 & $89.6 \%$ & $79.5 \%$ \\
Average & $98.9 \%$ & $82.3 \%$ \\
SD & 13.1 & 3.9 \\
RSD (\%) & 13.3 & 4.8 \\
\hline
\end{tabular}

TABLE IV

Recovery for Indian Mackerel Fish

\begin{tabular}{lr}
\hline Sample ID & $\begin{array}{c}\text { Recoveries } \\
\text { MtHg }\end{array}$ \\
\hline Indian Mackerel Fish Spike 1 & $86.3 \%$ \\
Indian Mackerel Fish Spike 2 & $96.2 \%$ \\
Indian Mackerel Fish Spike 3 & $83.2 \%$ \\
Indian Mackerel Fish Spike 4 & $109.1 \%$ \\
Indian Mackerel Fish Spike 5 & $95.4 \%$ \\
Average & $94.1 \%$ \\
SD & 10.1 \\
RSD (\%) & 10.8 \\
\hline
\end{tabular}

The method is quick and simple to set up, yet offers a repeatable and robust analysis, providing an ideal solution for the analysis of fish samples for potential mercury contamination. The detection limits of the various mercury species obtained with this system are low enough for mercury speciation of many real samples without requiring complicated sample pretreatment.

$\overline{\text { Received January 25, } 2011 .}$

\section{Atomic}

REFERENCES

1. United Nations Environmental Programme (2002) Global Mercury Assessment.

2. Food Safety Information Website, http://www.foodhaccp.com.

3. Commission Regulation (EC) No 1881/2006 of 19 December 2006: Setting maximum levels for certain contaminants in foodstuffs.

4. Codex General Standard for Contaminants and Toxins in Foods CODEX STAN 193-1995, Rev.3-2007.

5. P. Hajeb, S. Jinap, A. Ismail, A.B. Fatimah, B. Jamilah, M. Abdul Rahim, Food Control 20, 79 (2009).

6. Kyung Su Park, Jeong Sook Kim, HyoMin Lee, Heesoo Pyo, Sun-Tae Kim, Kang Bong Lee, Speciation of Six Arsenic Compounds in Korean Seafood Samples. Key Engineering Materials, 277-279, 431-437 (2005).

7. Chwei-Sheng Chiou, Shiuh-Jen Jiang, K. Suresh Kumar Danadurai, Spectrochim. Acta, Part B, 56, 1133 (2001).

8. R. Rai, W. Maher, F. Kirkowa, J. Anal. At. Spectrom, 12, 1560 (2002). 